\title{
MECHANSIMS FOR LATITUDINAL TRANSPORT OF ENERGETIC PARTICLES IN THE HELIOSPHERE
}

\author{
L. A. FISK ${ }^{1}$ AND J. R. JOKIPII ${ }^{2}$ \\ ${ }^{1}$ Dept. of Atmospheric and Space Sciences, University of Michigan, Ann Arbor, MI 48105, USA \\ ${ }^{2}$ Depts. of Planetary Sciences and Astronomy, University of Arizona, Tucson, AZ 85721, USA
}

Received: 5 November 1998; Accepted: 5 February 1999

\begin{abstract}
Energetic particles in the heliosphere, from relatively low-energy particles which are accelerated in Corotating Interaction Regions (CIRs) to galactic cosmic rays, are observed to propagate relatively easily in heliographic latitude. Two mechanisms for this transport appear possible: cross-field diffusion, or, in a recent model for the heliospheric magnetic field, by direct magnetic connection. The commonalties and differences of these two mechanisms are considered, and the need for future observations and modeling efforts are discussed.
\end{abstract}

\section{Introduction}

Observations of energetic particles and galactic cosmic rays from the Ulysses spacecraft have revealed the interesting fact that these particles appear to propagate in heliographic latitude with relative ease. Galactic cosmic rays show corotating variations, evidently produced by CIRs at low latitudes, up the highest latitudes seen by Ulysses (Simpson et al., 1996), where there was no evidence of corotating variations in the solar wind or magnetic field. CIRs form from the interaction of high and low speed solar wind flows. During the time period of Ulysses, these regions are confined to within about $40^{\circ}$ of the equatorial plane of the Sun, and are unlikely to expand much beyond this range in the more distant heliosphere. Even more interesting was the fact that very low energy electrons $(\approx 50 \mathrm{keV})$ and sub $\mathrm{MeV}$ protons, which are known to be accelerated at the shock waves which surround CIRs, are observed up to the highest latitudes viewed by Ulysses, up to $80^{\circ}$ (e.g., Lanzerotti et al., 1997).

These observations are particularly curious when we consider the standard form for the heliospheric magnetic field, which was shown by Parker (1958) to be an Archemedes spiral pattern, lying on cones of constant latitude. The field is assumed to be attached to the rotating Sun, and carried radially outward with the solar wind. In this model, then, there needs to be extensive cross-field propagation, which would not necessarily have been expected, particularly for the low energy particles.

There appear to be two choices to account for the Ulysses observations: there is extensive cross-field diffusion, as has been argued for by, e.g., Jokipii and Parker (1969), Kóta and Jokipii (1995), which relies on a randomly braided magnetic field, 
or the simple model for the heliospheric magnetic field of Parker (1958) needs to be modified, as has been suggested by Fisk (1996), into a model which allows direct magnetic connection from low to high latitudes. We consider each of these possibilities. It should be noted, however, that these models are not mutually exclusive. Each may be operative to some degree, and it will be necessary to determine the extent to which each possibility, or perhaps some other possibility, is dominant.

\section{Latitudinal Transport by Cross-Field Diffusion}

Consider first the diffusion of fast charged particles across the average magnetic field. In order for this to be adequate to explain the observations of the transport of CIR-generated particles to high latitudes, a substantially larger value of the ratio of perpendicular to parallel diffusion is required than would be expected by using classical scattering theory. If $\lambda_{\|}$is the parallel scattering mean free path and $r_{\mathrm{g}}$ is the particle gyroradius in the average magnetic field, then the classical expression is:

$$
\frac{\kappa_{\perp}}{\kappa_{\|}}=\frac{1}{1+\left(\lambda_{\|} / r_{\mathrm{g}}\right)^{2}},
$$

For typical values of $\lambda_{\|}$, this ratio is very small, of the order of $10^{-4}$ or less for low energy ions, which is too small to allow meaningful propagation of CIR particles to high latitudes.

More than 30 years ago it was pointed out that the random walk, or braiding, of the magnetic lines of force due to turbulence in the magnetic field could provide a large contribution to perpendicular diffusion (Jokipii, 1966; Jokipii and Parker, 1969). This concept has an intuitive interpretation. Particles follow individual field lines which are randomly braided on a variety of length scales. Since the field lines could be braided down to the scale of the particle gyroradius, it should be possible for particles to move from field line to field line, thereby propagating normal to the direction of the mean magnetic field. The actual braiding itself could have several causes. Jokipii and Parker (1969) and more recently Jokipii et al. (1995) related the braiding to observed supergranule motions in the photosphere, where the expected diffusion of field lines on the Sun should lead to considerable intertwining. This is illustrated in Fig. 1. It is also possible, as can be inferred from recent solar observations (Schrijver et al., 1997), that reconnection of the coronal magnetic field with emerging loop structures will cause the field lines to reorient their positions, thereby becoming intertwined with other field lines. Enhanced turbulence over the solar poles as predicted by Jokipii and Kóta (1989) and observed by Ulysses (Smith et al., 1995) is also a possibility for increased braiding. 


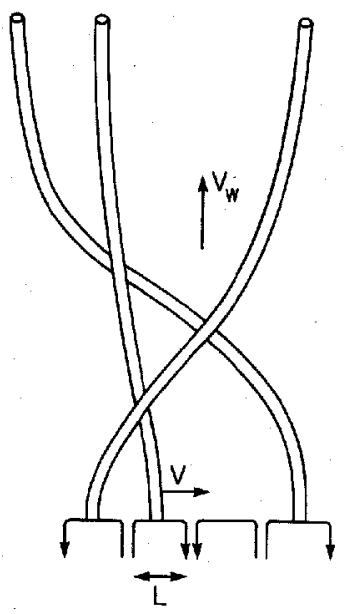

Figure 1. Schematic illustration of the introduction of field braiding near the Sun (Jokipii and Parker, 1969). The basic picture remains much the same if there is signficant reconnection near the Sun as presently thought.

Braiding by itself is not sufficient for effective cross field diffusion, but rather the particles must actually be able to move to different field lines. The extent to which that is possible, particularly for very low rigidity particles such as $50 \mathrm{keV}$ electrons, is not well understood (see, e.g., Giacalone, 1999). Figure 2 illustrates results from numerical simulations (Giacalone, 1999), in which a realistic random field is computed using plausible solar parameters, and a straightforward extrapolation into the heliosphere. For this case, and a small amount of ad hoc scattering which produces isotropy and motion across field lines, ratios of $\kappa_{\perp} / \kappa_{\|} \approx 0.02$ were obtained. This value produces considerable latitudinal transport. The random scattering alone would produce a much smaller $\kappa_{\perp} / \kappa_{\|}$, of the order of $10^{-4}$.

Such values of $\kappa_{\perp}$ are (and for many years have been) readily incorporated into existing global numerical simulations of cosmic-ray transport. It is found (e.g., Kóta and Jokipii, 1995) that ratios of $\kappa_{\perp} / \kappa_{\|} \approx .02-.05$ produce significant corotating effects to high latitudes. This is close to the value deduced by Giacalone (1999).

\section{Latitudinal Transport by Direct Magnetic Connection}

Consider next latitudinal transport by direct magnetic connection. As was pointed out by Fisk (1996), the interplay between the differential rotation of the photosphere, and the non-radial expansion of the solar wind from more rigidly rotating polar coronal holes can lead to large excursions of the heliospheric magnetic field in latitude. In this section, we review the model of Fisk (1996) and consider its consequences for the transport of energetic particles from low to high latitudes.

The field model of Fisk (1996) is based on three assumptions: The heliospheric magnetic field is attached to the photosphere, which differentially rotates. The high speed solar wind expands non-radially from polar coronal holes, i.e., the high speed solar wind originates from a limited volume near the base of the corona, and 

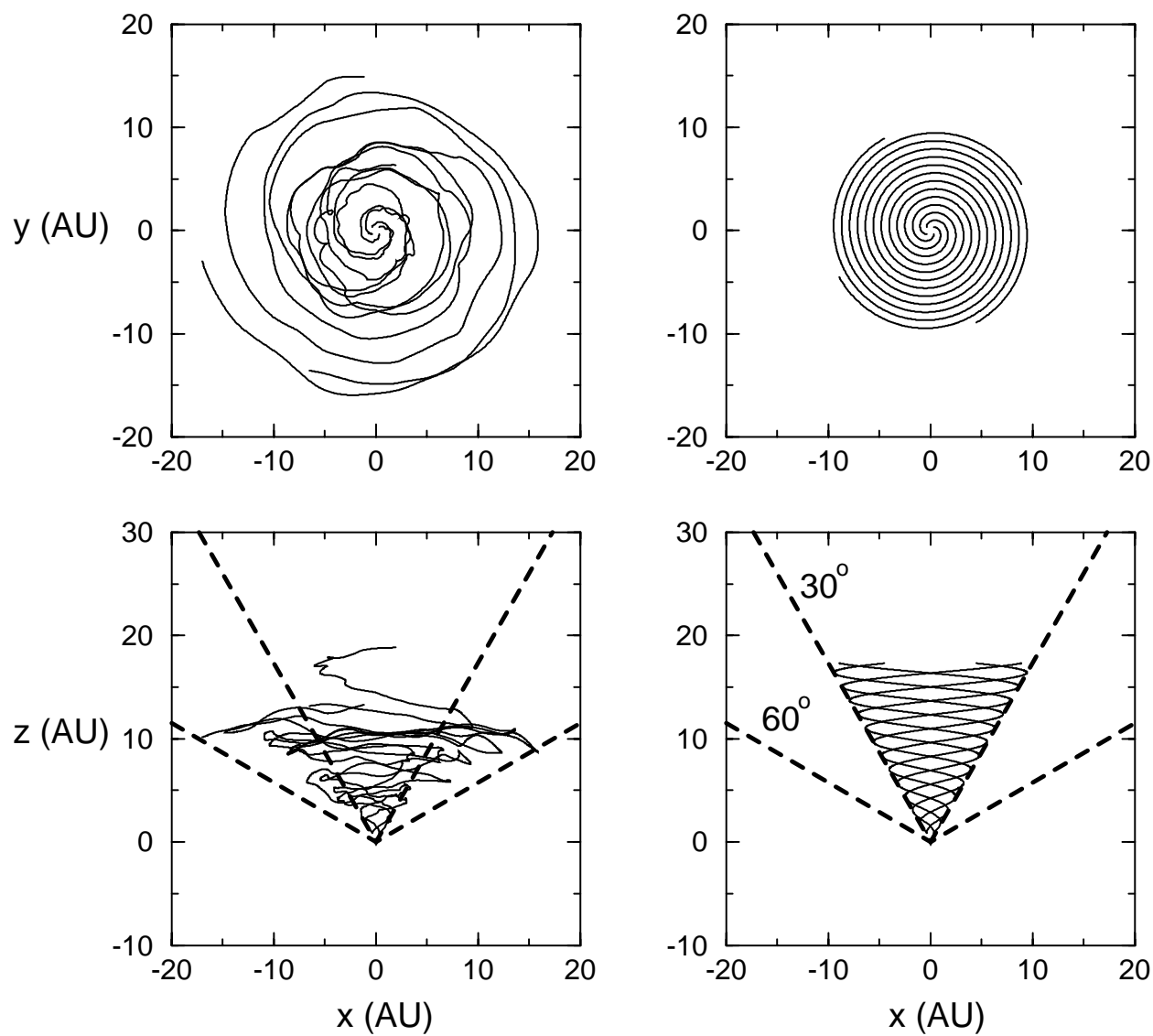

Figure 2. Illustration of the magnetic field lines as projected out into the heliosphere (Giacalone, 1999). The right two panels show two orthogonal views of the Parker spiral and the left two panels the corresponding views of the field with the random walk or braiding included. The results are for reasonable random walk parameters near the Sun's surface: a velocity of $0.6 \mathrm{~km} / \mathrm{s}$ and a time scale of one day.

expands to fill a significant volume of the heliosphere. The expansion of the solar wind in the polar coronal holes is about an axis that is both offset from the solar rotation axis and which tends to rotate rigidly at approximately the equatorial rotation rate. The last assumption is consistent with the observed property of coronal holes, that they tend to rotate rigidly (e.g., Bird and Edenhofer, 1990).

The consequences of these assumptions for the magnetic field in the corona are illustrated in Fig. 3, which is drawn in the frame of reference which corotates at the equatorial rotation rate. The axis $\mathbf{M}$ is the axis of symmetry of the expansion of the solar wind from the polar coronal hole. By the above assumptions it is offset from the rotation axis $\Omega$ and is fixed in this reference frame. Consider, then, the magnetic field line which originates from the heliographic pole. It undergoes a nonradial expansion about $\mathbf{M}$ and penetrates the outer surface in Fig. 3 at the location 


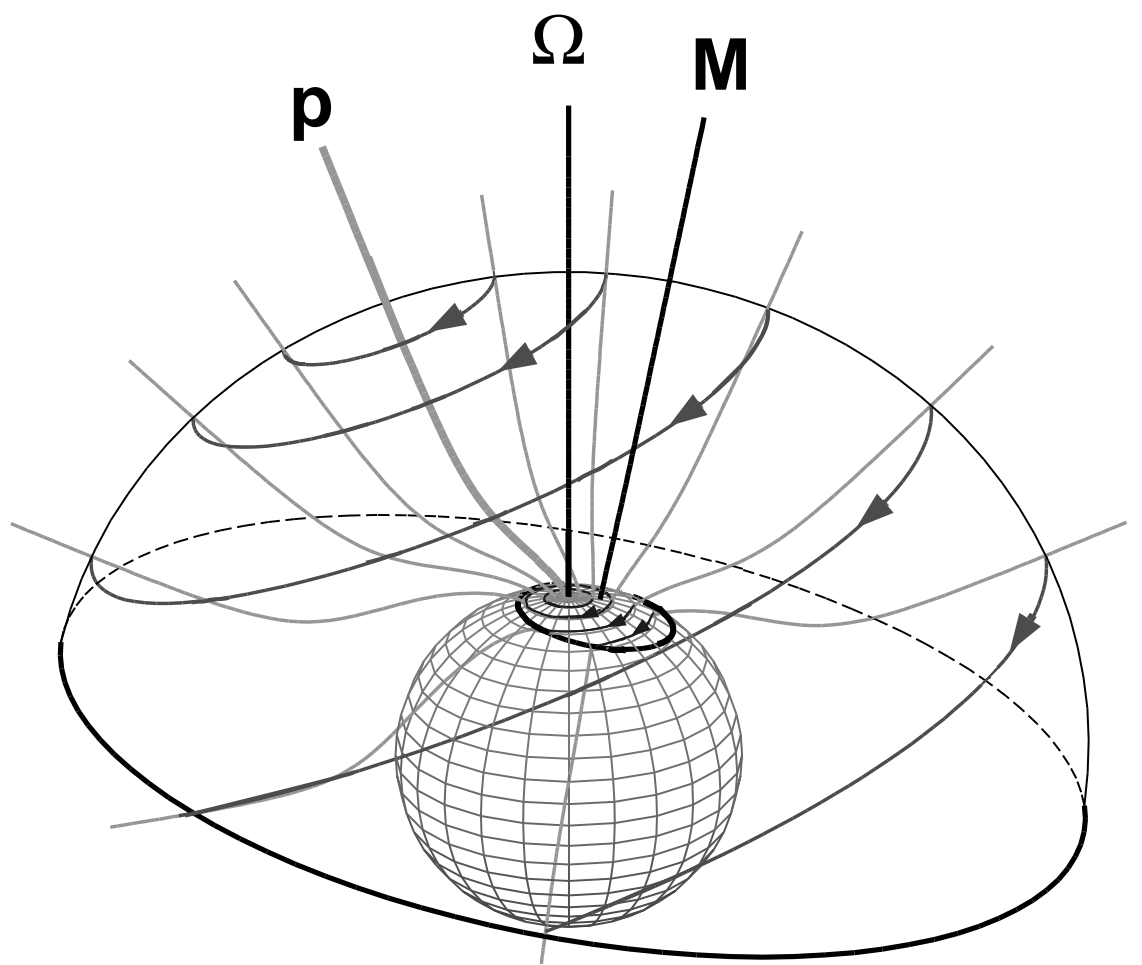

Figure 3. An illustration of the orientation of the magnetic field in the corona, and the trajectories of these field lines, as predicted in the model of Fisk (1996) (after Zurbuchen et al., 1997).

p. All other field lines are anchored in the photosphere which differentially rotates around $\mathbf{p}$. These field lines execute circular motion in the photosphere about $\Omega$, which, following the non-radial expansion, results in circular motion (presumably distorted circular motion) about p. The circles on the outer surface of Fig. 3 mark the location of the footpoints of heliospheric magnetic field lines, or equivalently, the footpoints of heliospheric field lines can, in this model, make large excursions in heliographic latitude.

The resulting field patterns in the heliosphere can be quite complicated as is illustrated in Fig. 4. The figure is drawn for field lines whose footpoints in the corona, following the non-radial expansion of the solar wind, are at a latitude of $70^{\circ}$ north. The field lines are drawn out to a distance of approximately $20 \mathrm{AU}$. In the lower drawing, where there is no footpoint motion, the field lines remain at $70^{\circ}$ north, and simply execute the standard Archemedes spiral pattern appropriate for this latitude. In the top figure, with footpoint motion, the field lines extend over a wide latitude range. A field line whose footpoint is currently at $70^{\circ}$ north was, in its past history, at other latitudes. The solar wind drags the field lines out radially and, when combined with these latitude excursions of the footpoints, creates the complicated pattern. Note, in particular, that field lines which in the inner heliosphere, 


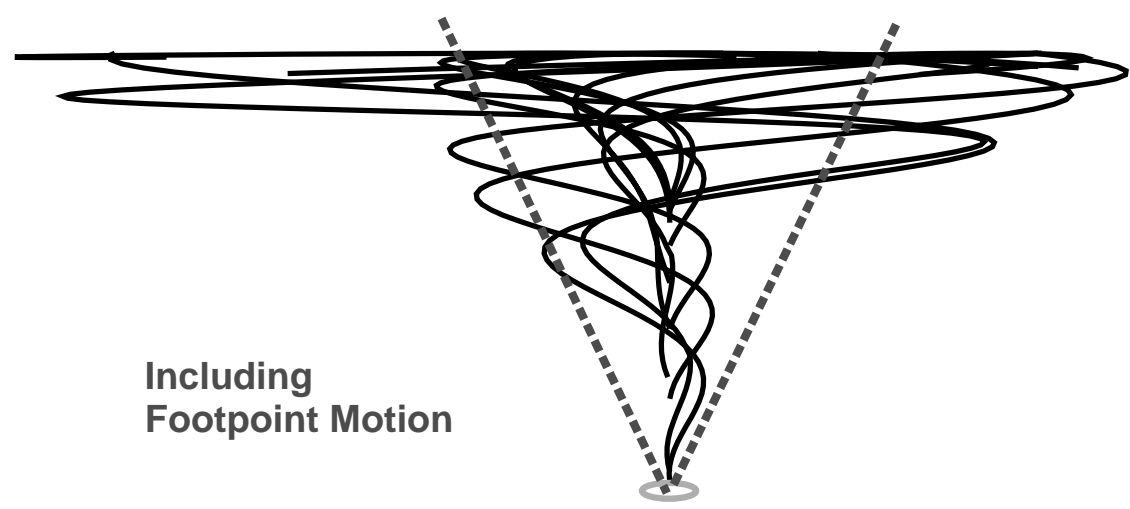

\section{Without \\ Footpoint Motion}

Figure 4. An illustration of the expected configuration of the heliospheric magnetic field comparing models in which there is footpoint motion, and in which there is no footpoint motion, respectively. The fields in both cases originate from $70^{\circ}$ north latitude. (after Fisk, 1996)

i.e. within a few $\mathrm{AU}$ of the Sun, are at $70^{\circ}$ north, connect to much lower latitudes within 15-20 AU of the Sun. In this model, then, there can be a direct magnetic connection from low to high latitudes in the heliosphere.

The two field configurations in Fig. 2, with and without footpoint motion, appear quite different when viewed on these large spatial scales, of more than $10 \mathrm{AU}$ However, when observed from a single spacecraft, the model with footpoint motion yields an orientation for the magnetic field which is always within about $10^{\circ}$, or less, of the orientation of the standard Parker field model without footpoint motion. With the large Alfvénic variations in the heliospheric magnetic field, particularly at high heliographic latitudes, it is thus hard to distinguish observationally between the two models, or equivalently to prove that the more complicated field model does in fact occur. 


\section{r [AU]}

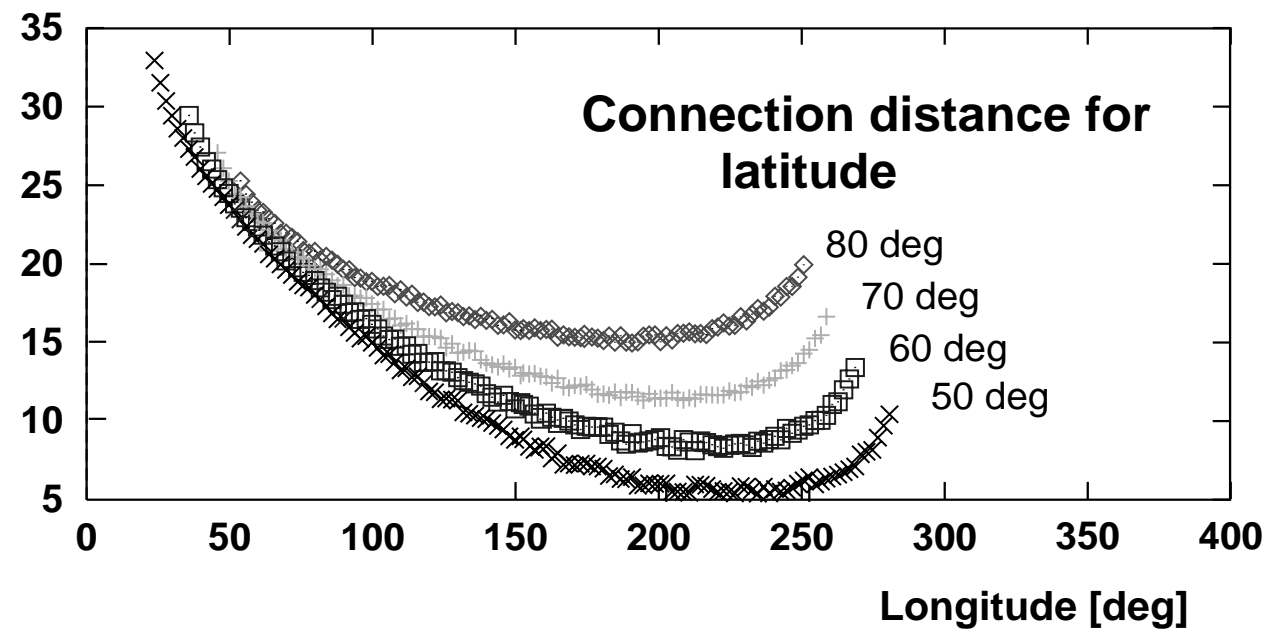

Figure 5. The heliocentric radial distance at which a field line from a given latitude and longitude crosses $30^{\circ}$ north latitude, i.e. enters the region where CIRs occur. The longitude scale is fixed on the rotating Sun, and of arbitrary phase. (after Zurbuchen et al., 1999)

However, there is some observational evidence in support of the model with footpoint motion. As can be seen in Fig. 3, at high latitudes, there are regions where the direction of the footpoint motion is opposite to that of the solar rotation. The heliospheric field originating from this region will thus be over-wound relative to the standard Archemedes spiral that would result from the equatorial rotation rate. Indeed, Forsyth et al. (1995) have shown that at high heliographic latitudes in the southern hemisphere the field is over-wound, which is difficult to account for by any other means since, for example, differential rotation by itself would lead to an under-wound field. Also, Zurbuchen et al. (1997) performed several observational tests on the heliospheric magnetic field, and although not conclusive, are consistent with the footpoint motion theory. They found that the field at high latitudes has two natural periodicities, one at about 20 days and the other at about 34 days. The latter is the expected high latitude rotation period, and the former is consistent with variations in the field following the footpoint trajectories in Fig. 3. Zurbuchen et al. (1997) also, through careful averaging, found a systematic variation in the polar component of the heliospheric magnetic field which in amplitude and phase is exactly as would be expected from the footpoint motion theory.

Consider, then, the extent to which the field model with footpoint motion can account for the ability of particles to transport from CIRs at low latitudes, up to high heliographic latitudes. Shown in Fig. 5, from Zurbuchen et al. (1999), are the heliocentric radial distances at which a field line from a given latitude and longitude on the Sun (following the non-radial expansion) will cross a heliocentric latitude of $30^{\circ}$, i.e. will enter into the region where CIRs occur. This calculation assumes 
a simple model for the footpoint motion in which the footpoint trajectories at the Sun are circles, as is described in Zurbuchen et al. (1997). For example, a field line which originates from $80^{\circ}$ in latitude and $50^{\circ}$ in longitude (the longitude coordinate system is fixed on the rotating Sun, and of arbitrary phase) will not connect to the CIR region until almost $30 \mathrm{AU}$ from the Sun. In contrast, a field line from $80^{\circ}$ in latitude and $200^{\circ}$ in longitude, connects within $15 \mathrm{AU}$. Note also that there is a longitude interval when there is no connection. The actual distance along the field line is of course much longer than the heliocentric radial distance, since the field follows the general spiral pattern. Within the first $15 \mathrm{AU}$ in radial distance from the Sun, the distance along the field is approximately twice the radial distance; at larger radial distances, the distance along the field is a larger multiple. Clearly, the connection distance is sufficiently short so that direct magnetic connection from low to high latitudes is possible.

\section{Concluding Remarks}

The two models for latitude transport - cross field diffusion by field line random walk and direct magnetic connection - share certain fundamental commonalties. In both cases, motion of the field lines at the Sun is required, and in both cases particle propagation is primarily along the field lines. In the case of field line random walk, the field lines move randomly at the Sun by supergranule motions or by reconnection. The motion of the field lines is then a statistical process. In the case of direct magnetic connection, the motion of the field lines at the Sun is systematic resulting from the interplay of the non-radial expansion of the solar wind, and the difference between the differential rotation of the photosphere and the more rigid rotation of coronal holes. Clearly, the two effects are not mutually exclusive, and both could occur simultaneously; however, with some mitigation of one effect on the other. If there is extensive random motion of field lines at the Sun, it will negate the impact of the systematic motions of the heliospheric field lines in latitude and the resulting direct connection to CIRs within the required 10-15 AU. Conversely, if there is direct magnetic connection so that field lines do connect to CIRs within 10-15 AU, then this should be a more expedient route for particles to take to reach high latitudes than by cross-field diffusion. In general the motion of particles along a single field line is likely to provide a shorter route for the particle transport in latitude than the motion along multiple field lines.

The issue of which effect, if either, is dominant can only be resolved observationally. Observations of the magnetic field are helpful but are unlikely to be conclusive. Observations at any one point in the solar wind cannot be expected to reveal the true nature of the intertwining of the magnetic field, and thus the opportunity for particles, particularly low rigidity particles, to move effectively from one field line to the next. Similarly, the systematic motions of the field lines, even with considerable motions of the footpoints near the Sun, will yield small 
and very difficult to discern differences between this field configuration and the standard Parker spiral field with no latitudinal component of the field. Rather, discriminating between the alternative explanations for latitude transport will require detailed models for particle propagation and the comparisons of these models with observations. Models with cross field diffusion are available as was described in Sect. 2; however, no numerical model is currently available which includes the full impact of a field resulting from systematic footpoint motions.

\section{Acknowledgements}

LAF was supported, in part, by NASA contracts NAG5-2810, NAG5-7111 and JPL contract 955460. JRJ supported, in part, by the National Science Foundation under Grant ATM 9616547 and by the National Aeronautics and Space Administration under Grants NAGW-4542, NAGW 1931. JRJ's research is also part of an IDS project on the Ulysses Mission JPL \# 960843.

\section{References}

Bird, M. K., and Edenhofer, P.: 1990, 'Remote Sensing Observations of the Solar Corona', in R. Schwenn and E. Marsch (eds.), Physics of the Inner Heliosphere, Springer-Verlag, Berlin, pp. $13-19$.

Burlaga, L.F., McDonald F. B., Goldstein, M. L., and Lazarus, A. L.: 1996, 'Cosmic Ray Modulation and Turbulent Interaction near 11 AU', J. Geophys. Res. 90, 12,027-12,039.

Fisk, L. A.: 1996, 'Motion of the Footpoints of Heliospheric Magnetic Field Lines at the Sun: Implications for Recurrent Energetic Particle Events at High Heliographic Latitudes', J. Geophys. Res. 101, 15,547-15,554.

Forsyth, R. J., Balogh, A., Smith, E. J., Murphy, N., and McComas, D. J.: 1995, 'The Underlying Magnetic Field Direction in Ulysses Observations of the Southern Polar Hemisphere', Geophys. Res. Lett. 22, 3321-3324.

Giacalone, J.: 1999, 'Particle Transport and Acceleration at Corotating Interaction Regions', Adv. Space Res. 23, 581-590.

Jokipii, J. R.: 1966, 'Particles in a Random Magnetic Field I', Astrophys. J. 146, 480.

Jokipii, J. R., and Parker, E. N.: 1969, 'Random Walk of Magnetic Lines of Force in Astrophysics', Phys. Rev. Lett. 21, 44.

Jokipii, J. R., and Kóta, J.: 1989, 'The Polar Heliospheric Magnetic Field', Geophys. Res. Lett. 16, $1-4$.

Jokipii, J. R., Kóta, J., Giacalone, J., Horbury, T. S., and Smith, E. J.: 1995, 'Interpretation and Consequences of Large-scale Magnetic Variances at High Heliographic Latitude', Geophys. Res. Lett. 22, 3385-3388.

Kóta, J., and Jokipii, J. R.: 1995, 'Corotating Variations of Cosmic Rays Near the South Heliospheric Pole', Science 268, 1024-1025.

Lanzerotti, L. J., MacLennan, C. G., Armstrong, T. P., Roelof, E. C., Gold, R.E., and Decker, R. B.: 1997, 'Low Energy Charged Particles in the High Latitude Heliosphere', Adv. Space Res. 19, 851-854. 
Parker, E. N.:1958, 'Dynamics of the Interplanetary Gas and Magnetic Field', Astrophys. J. 128, 664. Schrijver, C. J., Title, A. M., van Ballegooijen, A. A., Hagenaar, H. J., and Shine, R. A.: 1997, 'Sustaining the Quiet Photospheric Network: The Balance of Flux Emergence, Fragmentation, Merging, and Cancellation', Astrophys. J. 487, 424-436.

Simpson, J. A., Zhang, M., and Bame, S.: 1996, 'A Solar Polar North-south Asymmetry for Cosmicray Propagation in the Heliosphere', Astrophys. J. 465, L69-L72.

Smith, E. J., Neugebauer, M., Balogh, A., Bame, S. J., Lepping, R. P., and Tsurutani, B. T.: 1995, 'Ulysses Observations of Latitude Gradients in the Heliospheric Magnetic Field: Radial Component and Variances', Space Sci. Rev. 72, 165-170.

Zurbuchen, T.H., Schwadron, N. A., and Fisk, L. A.: 1997, 'Direct Observational Evidence for a Heliospheric Magnetic Field with Large Excursions in Latitude', J. Geophys. Res. 102, 24,175.

Zurbuchen, T.H., Fisk, L. A., Schwadron, N. A., and Pizzo, V.: 1999, 'A Simple Model for the new Heliospheric Field Configuration and the High-Latitude Transport of Low-Energy Particles', $J$. Geophys. Res. in press.

Address for Offprints: J. R. Jokipii, The University of Arizona, Departments of Planetary Sciences and Astronomy, Tucson, AZ 85721-9200, U.S.A. 